\title{
Christiane M. Thomsen, Burchards Bericht über den Orient
}

\section{Stéphane Péquignot}

\section{OpenEdition}

\section{Journals}

Édition électronique

URL : http://journals.openedition.org/ifha/10150

DOI : 10.4000/ifha. 10150

ISSN : 2198-8943

\section{Éditeur}

IFRA - Institut franco-allemand (sciences historiques et sociales)

\section{Référence électronique}

Stéphane Péquignot, « Christiane M. Thomsen, Burchards Bericht über den Orient », Revue de l'IFHA [En ligne], Date de recension, mis en ligne le 21 juin 2019, consulté le 24 septembre 2020. URL : http:// journals.openedition.org/ifha/10150 ; DOI : https://doi.org/10.4000/ifha.10150

Ce document a été généré automatiquement le 24 septembre 2020

(C)IFHA 


\section{Christiane M. Thomsen, Burchards Bericht über den Orient}

\section{Stéphane Péquignot}

\section{RÉFÉRENCE}

Christiane M. Thomsen, Burchards Bericht über den Orient. Reiseerfahrungen eines staufischen Gesandten im Reich Saladins 1175/1176, Berlin/Boston : Walter de Gruyter (Europa im Mittelalter - Abhandlungen und Beiträge zur historischen Komparatistik, hrsg. von Michael Borgolte und Wolfgang Huschner, 29), 2018, 654 p., 109,95€ 
Dans un texte dont l'original est perdu, mais qui est repris par huit témoins antérieurs au milieu du XIII ${ }^{\mathrm{e}}$ siècle, un certain Burchard, vicedominus de Strasbourg, prétend avoir reçu de l'empereur Frédéric I ${ }^{\mathrm{er}}$ Barberousse l'ordre d'effectuer une ambassade (legatio) auprès de Saladin. Il développe à la suite de cette affirmation liminaire le récit d'un périple en Méditerranée. Trois questions principales guident Christiane M. Thomsen dans l'analyse de ce texte : est-il composé à l'occasion d'un voyage d'ambassade? Les expériences décrites sont-elles celles de l'auteur? À quel genre, si le terme est pertinent, l'écrit de Burchard peut-il être rattaché ?

L'étude porte d'abord sur les formes et les

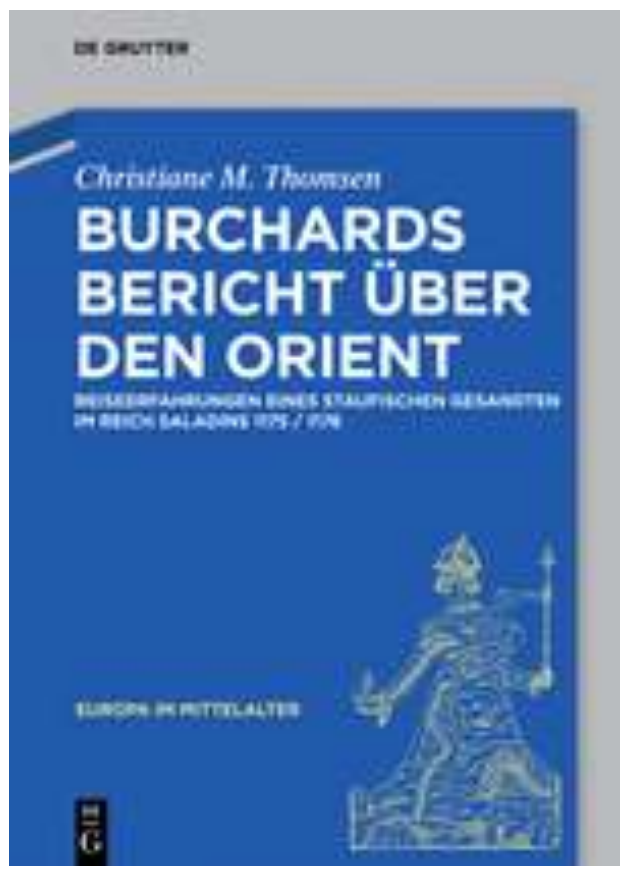
fonctions du récit. Écrivant dans un latin à la rhétorique modeste, Burchard suit un itinéraire qui le mène de Gênes en Égypte et en Syrie, le voyage du retour donnant lieu à des développements beaucoup plus succincts. L'auteur est extrêmement attentif à la géographie, aux villes et aux productions des territoires qu'il traverse. Il s'attache également à la description des us et coutumes des populations, avec une prédilection pour les musulmans. Il n'hésite pas, pour faciliter la compréhension à recourir aux comparaisons avec les réalités occidentales - le Nil est mis en regard avec le Rhin, les crocodiles, avec les cochons. De manière moins anecdotique, ses descriptions se distinguent par leur relative systématicité.

C. M. Thomsen envisage ensuite le rapport du texte avec le réel et l'actualité. D'une confrontation méticuleuse avec les sources latines disponibles, et les sources arabes le cas échéant, se dégage un bilan contrasté. En dépit de certaines oppositions assez rigides, par exemple entre la Corse qualifiée de terra sana et la Sardaigne de terra infirmissima, Burchard s'avère globalement bien informé. Il livre ainsi une vision détaillée d'Alexandrie où, tout en postulant des liens pour le moins douteux entre le Nil et l'Euphrate, et en faisant peu de cas de la variété des populations chrétiennes, la cité apparaît remarquable par sa taille, le nombre de ses habitants, son dynamisme économique et... son relatif délabrement. Ses descriptions du Caire, de Fustat, de Matariyya ne semblent pas reposer sur un savoir livresque préliminaire. En Syrie, il évoque Damas, le monastère de Saydnâyâ, dont l'icône de Marie suscite une vénération partagée par des pèlerins d'origines diverses, et les "assassins ", qu'il nomme heyssessini. C. M. Thomsen réévalue la validité, débattue par les spécialistes, des affirmations de Burchard à ce dernier sujet. Les passages très substantiels consacrés aux musulmans ne se limitent pas aux seuls aspects rituels ou religieux, et s'écartent remarquablement des stéréotypes alors dominants. De manière plus générale, Burchard se révèle assez indépendant des sources latines. Observateur perspicace, il rejoint sur de nombreux aspects des textes composés par des auteurs arabes. Son originalité réside notamment dans la description fine d'une Égypte contemporaine, alors que le pays ne retient guère l'attention dans les écrits des Latins avant le XIII ${ }^{\mathrm{e}}$ 
siècle. L'hypothèse de la mise à profit de sources orales, d'informateurs et d'intermédiaires, emporte alors l'adhésion.

La véracité du récit ainsi mise en perspective, l'ambassade est replacée dans l'histoire des relations entre les Staufen et les Ayyoubides. L'entreprise est rendue délicate par l'absence de toute mention externe à la mission de Burchard. Suite à l'établissement d'une première alliance entre Barberousse et Saladin en 1172-1174, la mission de l'envoyé impérial a très probablement lieu en 1175-1176. Le mobile n'en est pas précisé. À la volonté mutuelle de s'opposer aux projets byzantins, traditionnellement avancée par les historiens, C.M. Thomsen ajoute d'autres facteurs explicatifs importants : la puissance accrue de Saladin, dont l'autorité, maintenue sur l'Égypte, s'exerce désormais aussi sur la Syrie et le Yémen; la fragilisation de l'autorité de Barberousse en Italie, rendant plus nécessaire encore la lutte contre la formation d'une dangereuse alliance ennemie ; sa volonté enfin d'avoir l'honneur de protéger les lieux saints. À l'aune d'une mission qui reste nimbée d'un certain mystère, la personne même de Burchard ne sort que partiellement de l'ombre. Si son rôle de vicedominus n'est pas disputé, et trouve même un écho dans d'autres sources contemporaines sur l'évêché de Strasbourg, son identification avec un notaire impérial, proposée il y a quelques années par Alfred Haverkamp, est ici remise en cause au bénéfice du rapprochement avec un chapelain homonyme.

Quoi qu'il en soit de la trajectoire de son auteur, le récit connaît une réception notable. Selon des voies d'appropriation difficiles à suivre, le premier à s'en saisir est Arnold von Lübeck, dans sa Cronica. Thietmar l'annexe ensuite à sa Peregrinatio, et il figure également dans le Tractatus de locis et statu sanctae Ierosolomitanae, avant d'être repris par Jacques de Vitry à l'occasion de la cinquième croisade. Ce moment est décisif pour la diffusion du texte. Utilisé à des fins diverses, il apparaît alors comme un témoin authentique et fiable (p. 466-467).

À l'issue d'un commentaire mené avec une grande acribie, C. M. Thomsen parvient à établir de manière convaincante que le récit de Burchard prend place à un moment crucial d'inflexion dans les relations entre Frédéric $\mathrm{I}^{\mathrm{er}}$ Barberousse et Saladin. L'historicité, la qualité et l'originalité du témoignage livré par un observateur avisé ressortent dans une lumière nouvelle. Seuls certains éléments avancés pour déterminer la nature du "rapport d'ambassade " (Gesandtschaftsbericht) - une part mineure dans l'ouvrage - pourront laisser plus perplexe. L'approche typologique, rendue délicate par l'absence d'autres témoins contemporains, aurait ainsi sans doute gagné à une comparaison plus large avec des écrits de nature proche, tels ceux réalisés deux siècles auparavant par Liutprand de Crémone. Le silence sur les rencontres diplomatiques et les personnes contribuerait d'autre part selon $\mathrm{C}$. $\mathrm{M}$. Thomsen à prouver que le texte est un rapport d'ambassade effectué sur commande, complété par une relation orale. Estce l'argument le plus pertinent? Enfin, l'affirmation selon laquelle les rapports d'ambassade comporteraient à la fin du Moyen Âge deux parties, l'une sur les affaires diplomatiques, l'autre sur les pays, mérite d'être nuancée. Les cas vénitiens et anglais n'ont pas, en la matière, valeur universelle.

Ce sont là des détails. On retiendra plutôt que l'auteur met à disposition, outre l'édition d'un texte latin clairement établi avec les variantes disponibles, une étude très solide (98 pages de bibliographie en caractères serrés, nourrissant des notes de bas de page superlatives), enthousiaste et engagée sur un texte lisse en apparence seulement, en réalité fort complexe, énigmatique même à certains égards. La lecture de cette thèse 
soutenue à Berlin en 2016 intéressera dès lors tout particulièrement les historiens des territoires concernés (Corse, Sardaigne, Égypte, Syrie, Terre sainte dans une moindre mesure), les spécialistes des récits de voyage, de la transmission des manuscrits et des relations diplomatiques.

INDEX

Index chronologique : Moyen Âge

Thèmes : Histoire des États et des pouvoirs ; Histoire religieuse ; Histoire sociale ; Histoire des villes et des régions ; Histoire de la culture ; Sources

\section{AUTEURS}

STÉPHANE PÉQUIGNOT

EPHE, PSL, équipe SAPRAT 